\title{
SOLUBILIDADE DO SrO EM NaCl-KCl FUNDIDO A $7^{\circ} \mathrm{C}$
}

Richard Louis Combes

Institut Galilée - Université de Paris - Nord - F-93430 - Villetaneuse - França

Sérgio Luiz Koeller

Departamento de Química - ICE - Universidade Federal Rural do Rio de Janeiro - 23851-970 - Seropédica - RJ

Recebido em 19/10/98; aceito em 26/8/99

\begin{abstract}
STRONTIUM OXIDE SOLUBILITY IN MOLTEN NaCl-KCl AT $727^{\circ} \mathrm{C}$.The solubility product value of $\mathrm{SrO}$ has been found to be equal to $10^{-4,2}$ (molality scale) in molten equimolar mixture of $\mathrm{NaCl}$ and $\mathrm{KCl}$ at $727^{\circ} \mathrm{C}$, using a potentiometric method involving a calcia stabilized zirconia membrane electrode. This value, which is in a logical agreement with other alkaline-earth oxide determined solubilities, is compared to those of $10^{-5,8}, 10^{-3,0}$ and $10^{-3,08}$ (molality scale) found in the litterature 33, 22 and 5 years ago, respectively. Such discrepencies have called the attention of the authors, their possible reasons (methodology, titrating agent) are analyzed and a theoretical discussion, for considering the authors' value as more reliable, is given in this paper.
\end{abstract}

Keywords: strontium oxide; stabilized zirconia; molten salts.

\section{INTRODUÇÃO}

Vários cloretos alcalino terrosos são utilizados em processos eletrolíticos em sais fundidos, seja pela produção do próprio metal ( $\mathrm{Ba}, \mathrm{Sr}, \mathrm{Ca}$ e $\mathrm{Mg}$ ), ou como coadjuvante para ajustar a condutividade, a densidade ou a acidez do banho $\left(\mathrm{BaCl}_{2}\right.$, $\mathrm{CaCl}_{2}$ ). Segundo o conceito de oxoacidez desenvolvido por Lux $^{1}$ e em seguida por Flood e Førland ${ }^{2}$, os óxidos alcalino terrosos MO são a base conjugada dos cátions $\mathrm{M}^{2+}$ dos respectivos cloretos dissolvidos no sal fundido, e de acordo com as equações de dissociação:

$\mathrm{MCl}_{2}($ sólido $) \rightarrow \mathrm{M}^{2+}($ solução $)+2 \mathrm{Cl}^{-}$(solução),

$\mathrm{M}^{2+}($ solução $)+\mathrm{O}^{2-}$ (solução) $\rightarrow \mathrm{MO}$ (sólido),

é natural que a solubilidade dos óxidos alcalino terrosos em cloretos fundidos, tenha chamado a atenção dos químicos nos últimos $40 \operatorname{anos}^{3,4,5,6}$.

$\mathrm{Na}$ década de 60, Naumann e Reinhard ${ }^{7}$, usando a técnica de saturação e coleta de amostra, fizeram a determinação do produto de solubilidade do $\mathrm{BaO}, \mathrm{CaO}$ e SrO. Posteriormente, no final da década de 70 , Combes e colaboradores ${ }^{8,9}$ determinaram os valores dos produtos de solubilidade do $\mathrm{CaO}$, do $\mathrm{BaO}$ e do $\mathrm{MgO}$ na mistura equimolar fundida de $\mathrm{NaCl}$ e $\mathrm{KCl}$ a $727^{\circ} \mathrm{C}$. Esses resultados foram obtidos por titulação potenciométrica com um eletrodo de zircônia estabilizada com óxido de cálcio, empregado como indicador da concentração dos íons $\mathrm{O}^{2-}$ no banho fundido, conforme demonstrado nos trabalhos anteriores de Combes e colaboradores ${ }^{10,11,12}$.

Também no final da década de 70 , Delimarsky e Shapoval ${ }^{13}$ determinaram o produto de solubilidade do $\mathrm{CaO}$ e $\mathrm{MgO}$, e mais recentemente Cherginets e Khailova ${ }^{14}$, utilizando a mesma técnica potenciométrica, determinaram os valores do produto de solubilidade de uma série de óxidos alcalino terrosos no $\mathrm{NaCl}$ $\mathrm{KCl}$ a $700^{\circ} \mathrm{C}$.

\section{PARTE EXPERIMENTAL}

\section{Equipamento experimental utilizado}

1 - Forno elétrico tipo poço com sistema de controle de aquecimento.
2 - Reator em aço inox (317 L) protegido internamente contra corrosão por uma camada de alumina, na parte superior possui um colar de circulação de água para manter a refrigeração.

3 - Cadinho de alumina de alta pureza contendo o sal fundido dentro do reator.

4 - Eletrodo referência de primeira espécie $\mathrm{Ag} / \mathrm{AgCl}$, composto de um fio de prata em contato com uma solução 0,75 $\mathrm{mol} / \mathrm{kg}$ de $\mathrm{AgCl}$ em NaCl-KCl, contido num tubo de alumina porosa para manter o contato elétrico.

5 - Eletrodo indicador de $\mathrm{pO}^{2-}$ feito com um tubo de zircônia estabilizada, contendo em seu interior um fio de níquel em contato com uma mistura de níquel e óxido de níquel em pó. Os ítens 4 e 5 estão descritos no trabalho ${ }^{11}$. 6 - Termopar protegido do sal fundido por um tubo de alumina refratária.

Todos os produtos químicos usados foram "P.A." A mistura $\mathrm{NaCl}-\mathrm{KCl}$ foi preparada conforme técnicas de trabalho descritos em artigo ${ }^{6}$. Os reagentes $\mathrm{BaO} \mathrm{SrCl}_{2}$ foram previamente secos a vácuo a $120^{\circ} \mathrm{C}$ por 24 horas.

\section{Método experimental}

A determinação da constante de solubilidade (Ks) do $\mathrm{SrO}$ foi realizada através da reação oxoacidobásica do cátion $\mathrm{Sr}^{2+}$ com o ánion $\mathrm{O}^{2-}$, na mistura equimolar $\mathrm{NaCl}-\mathrm{KCl}$ fundida a $727^{\circ} \mathrm{C}$, de acordo com a seguinte reação:

$\mathrm{Sr}^{2+}$ (solução) $+\mathrm{O}^{2-}$ (solução) $\rightarrow \mathrm{SrO}$ (precipitado),

cuja expressão da constante de solubilidade é:

$\mathrm{Ks}_{(\mathrm{SrO})}=\left[\mathrm{Sr}^{2+}\right]\left[\mathrm{O}^{2-}\right]$

$\mathrm{Na}$ determinação do $\mathrm{Ks}$ do $\mathrm{SrO}$ utilizou-se o método da titulação potenciométrica do íon óxido $\left(\mathrm{O}^{2-}\right)$, usando um eletrodo de zircônia estabilizada ${ }^{28,9,10,11,12}$, seletivo aos íons $\mathrm{O}^{2-}$. $\mathrm{O}$ potencial medido pelo eletrodo obedece a expressão (5) com a concentração do íon $\mathrm{O}^{2-}$ expressa em molalidade. Blander ${ }^{15}$ mostrou que para frações molares menores que 0,01 , pode-se descrever a atividadede dos íons $\mathrm{O}^{2-}$ através da fração molar ou qualquer outra unidade de concentração. Neste trabalho adotou-se a escala molal: 
$\mathrm{E}=\mathrm{E}^{\mathrm{o}}-2,3 \mathrm{RT} / 2 \mathrm{~F} \log \left[\mathrm{O}^{2-}\right]$

$-\log \left[\mathrm{O}^{2-}\right]=\mathrm{pO}^{2-}$

Inicialmente, preparou-se a mistura eutética $\mathrm{NaCl}-\mathrm{KCl}$ com $\mathrm{SrCl}_{2}$, previamente seco, que foi colocada em um cadinho de alumina $99,9 \%$ dentro do reator. A célula foi fechada e submetida a vácuo de $0,01 \mathrm{~atm}$, durante o aquecimento, até a temperatura de $400^{\circ} \mathrm{C}$. No processo de aquecimento foi utilizado um controlador de temperatura, cuja taxa foi de $120^{\circ} \mathrm{C}$ por hora, até alcançar a temperatura de $700^{\circ} \mathrm{C}$, a partir da qual a temperatura do banho de sal fundido foi controlada com um Variac e mantida a $727 \pm 1^{\circ} \mathrm{C}$.

$\mathrm{A}$ adição do íon $\mathrm{O}^{2-}$ foi feita através de quantidades conhecidas de $\mathrm{BaO}$ (previamente seco em estufa a $120^{\circ} \mathrm{C}$ por 24 horas), que, em trabalho anterior realizado por Combes e colaboradores $^{9}$, mostrou ser uma oxobase forte. Em seguida à titulação, foi adicionado $\mathrm{BaO}$ em excesso, até atingir o ponto de saturação do $\mathrm{BaO}$, o que permitiu a calibração do eletrodo indicador, já que no ponto de saturação a constante de solubilidade é dada pela equação:

$\mathrm{Ks}_{(\mathrm{BaO})}=\left[\mathrm{Ba}^{2+}\right]\left[\mathrm{O}^{2-}\right]$

e pela estequiometria: $\left[\mathrm{Ba}^{2+}\right]=\left[\mathrm{O}^{2-}\right]$, então $\mathrm{pO}^{2-}=(\mathrm{pKs})^{1 / 2}$.

Fez-se, novamente, a calibração do eletrodo para confirmar o ponto de saturação obtido e para verificar se o potencial obedeceu a expressão:

$\mathrm{E}=\mathrm{E}^{\mathrm{o}}-\mathrm{k} \log [\mathrm{BaO}]$

com $\mathrm{E}^{\mathrm{o}}$ constante, $\mathrm{k}=2,3 \mathrm{RT} / 2 \mathrm{~F} \approx 0,099 \mathrm{~V}$ a $1000 \mathrm{~K}$ e $[\mathrm{BaO}]$ é a concentração expressa na unidade de molalidade.

\section{RESULTADOS}

Os valores do potencial obtidos durante a titulação do $\mathrm{Sr}^{2+}$ pelos íons $\mathrm{O}^{2-}$ estão na Tabela 1 e foram relacionados com grau de avanço $(\alpha)$. Por definição, $\alpha$ é a razão entre as concentrações de $\mathrm{BaO}$ adicionada e de $\mathrm{SrCl}_{2}$ inicial e igual a $0,054 \mathrm{~mol} / \mathrm{kg}$.

Tabela 1. Resultados experimentais da titulação do $\mathrm{Sr}^{2+}$ e da padronização do eletrodo indicador

\begin{tabular}{cccccc}
\hline \multicolumn{3}{c}{ Titulação } & \multicolumn{3}{c}{ Padronização } \\
\hline -E (mV) & $\alpha$ & $\mathrm{pO}^{2-}$ & $-\mathrm{E}(\mathrm{mV})$ & $\begin{array}{c}-\log [\mathrm{BaO}] \\
(\mathrm{mol} / \mathrm{kg})\end{array}$ & $\mathrm{pO}^{2-}$ \\
\hline 636 & 0,30 & 2,9 & 612 & 2,95 & 2,9 \\
644 & 0,50 & 2,7 & 638 & 2,70 & 2,7 \\
648 & 0,60 & 2,6 & 678 & 2,30 & 2,3 \\
668 & 0,78 & 2,4 & 708 & 2,00 & 2,0 \\
683 & 0,88 & 2,2 & 730 & 1,79 & 1,8 \\
698 & 0,99 & 2,1 & 752 & 1,57 & 1,6 \\
716 & 1,10 & 1,9 & 776 & 1,31 & 1,3 \\
721 & 1,20 & 1,9 & 790 & 1,20 & 1,2 \\
736 & 1,30 & 1,7 & 794 & 1,15 & 1,15 \\
744 & 1,40 & 1,6 & 798 & 1,10 & 1,15 \\
771 & 1,50 & 1,5 & 796 & 1,05 & 1,15 \\
776 & 1,70 & 1,4 & 796 & 1,00 & 1,15 \\
\hline
\end{tabular}

A relação entre $\mathrm{pO}^{2-}$ e $\alpha$ está representada na equação (13) e foi obtida a partir do balanço de massa da reação oxoacidobásica descrita na equação 3 , onde:

$\left[\mathrm{Sr}^{2+}\right]=\left[\mathrm{Sr}^{2+}\right]_{0}-[\mathrm{SrO}]$,

$\left[\mathrm{O}^{2-}\right]=[\mathrm{BaO}]-[\mathrm{SrO}]$,
$\alpha=[\mathrm{BaO}] /\left[\mathrm{Sr}^{2+}\right]_{\mathrm{o}}$,

e substituindo as equações 9, 10 e 11 na equação 4, chegandose à seguinte expressão:

$\mathrm{Ks}=\left[\mathrm{O}^{2-}\right](1-\alpha)\left[\mathrm{Sr}^{2+}\right]_{\mathrm{o}}+\left[\mathrm{O}^{2-}\right]^{2}$

Resolvendo-se a equação (12) e aplicando a definição de $\mathrm{pO}^{2-}$, tem-se:

$\mathrm{pO}^{2-}=-\log \frac{\left[\mathrm{SrCl}_{2}\right]_{\mathrm{o}}(\alpha-1)+\sqrt{\left(\left[\mathrm{SrCl}_{2}\right]_{\mathrm{o}}(\alpha-1)\right)^{2}+4 \mathrm{Ks}}}{2}$

Para representar a equação (13) no diagrama de $\mathrm{pO}^{2-}$ versus $\alpha$ atribui-se valores para Ks na equação, e desta forma obtemse a curva que melhor descreve os pontos experimentais, conforme metodologia de trabalhos anteriores ${ }^{9,10}$.

$\mathrm{O}$ valor de $\mathrm{Ks}=6,3 \times 10^{-5}$ representa a curva pontilhada, o que possibilitou a determinação do $\mathrm{pKs}$ do $\mathrm{SrO}$, cujo valor encontrado foi $4,2 \pm 0,1$, na escala de molalidade. A representação da curva e os valores experimentais estão na Figura 1.

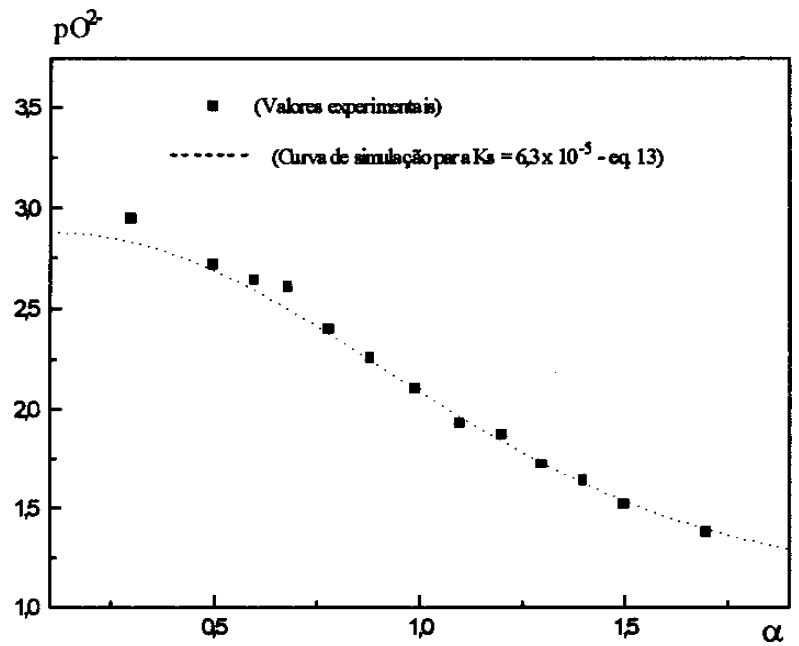

Figura 1. Curva de simulação e valores experimentais da titulação do $\mathrm{Sr}^{2+}$ pelo $\mathrm{BaO}$.

$\mathrm{O}$ eletrodo foi padronizado com $\mathrm{BaO}$, repetindo o trabalho realizado por Combes e Andrade ${ }^{9}$. Os valores do potencial do eletrodo indicador de $\mathrm{pO}^{2-}$ em função do $\mathrm{BaO}$ adicionado (escala de molalidade), estão descritos na Tabela 1 e ilustrados na Figura 2. A reta, cujo coeficiente angular é igual a $-0,1 \mathrm{~V}$, representa a solubilidade do $\mathrm{BaO}$ e a linha horizontal corresponde à precipitação. Estes resultados confirmam os valores obtidos e demonstram a padronização do eletrodo indicador.

\section{DISCUSSÃO}

O valor do produto de solubilidade obtido neste trabalho é de $4,2 \pm 0,1$, na escala de molalidade, a $727{ }^{\circ} \mathrm{C}(1000 \mathrm{~K})$. Como mostrado na Tabela 2 , este valor é bastante menor que 5,8 , pKs obtido por Naumann e Reinhard ${ }^{7}$, os primeiros que fizeram a determinação. Os resultados obtidos por Combes e colaboradores e o encontrado neste trabalho, se comparados aos de Naumann, são menores, na mesma ordem de grandeza. Porém, tanto o resultado deste trabalho quanto o de Combes são mais confiáveis, por terem sido determinados pelo método de titulação potenciométrica no próprio sal fundido ${ }^{8,9}$.

Ao contrário, os valores encontrados por Delimarsky e Shapoval ${ }^{13}$ e mais recentemente, por Cherginets e Khailova ${ }^{14}$, são menores, apesar de determinados pela mesma técnica 


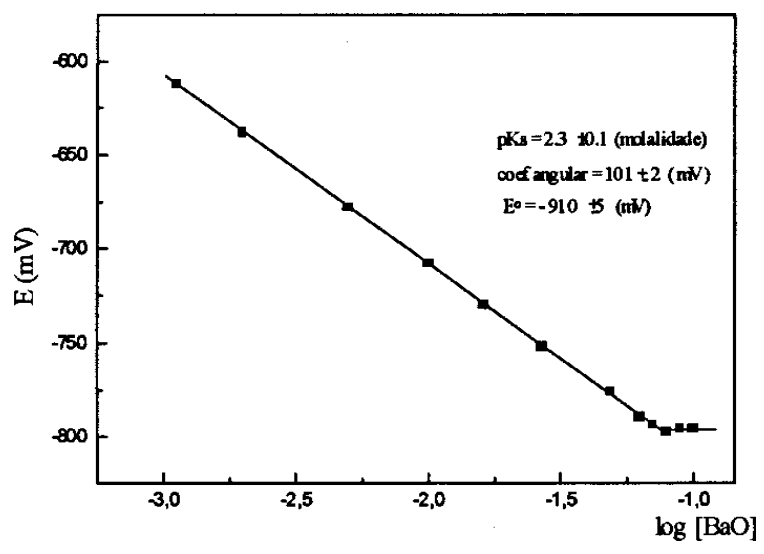

Figura 2. Curva de padronização do eletrodo indicador de $p \mathrm{O}^{2-}$.

Tabela 2. Relação dos produtos de solubilidades dos alcalinos terrosos (escala de molalidade) no $\mathrm{NaCl}-\mathrm{KCl}$ fundido a $727^{\circ} \mathrm{C}$.

\begin{tabular}{lcccc}
\hline Autores & $\mathrm{BaO}$ & $\mathrm{SrO}$ & $\mathrm{CaO}$ & $\mathrm{MgO}$ \\
\hline Combes $^{8,9}$ & 2,31 & - & 5,0 & 9,0 \\
Naumann $^{7}$ & 4,1 & 5,8 & 6,5 & - \\
Delismarsky $^{13}(*)$ & - & 3,0 & - & 8,46 \\
Cherginets $^{14}(*)$ & 2,30 & 3,08 & 4,36 & 9,27 \\
Este trabalho & - & 4,2 & - & - \\
\hline
\end{tabular}

(*) $700^{\circ} \mathrm{C}$

potenciométrica. Uma explicação para a diferença é o fato desses autores terem usado o $\mathrm{NaOH}$ como oxobase para a titulação, e terem considerado a mesma, como totalmente dissociada (oxobase forte).

Combes e colaboradores ${ }^{12}$ demonstraram que o íons $\mathrm{HO}^{-}$ estão envolvidos no seguinte equilíbrio:

$$
2 \mathrm{HO}^{-}(\text {solução }) \rightarrow \mathrm{H}_{2} \mathrm{O} \text { (vapor) }+\mathrm{O}^{2-} \text { (solução), }
$$

que necessariamente impõe o controle de uma nova variável, a pressão de vapor da água. O valor da constante da reação acima é $10^{-2,6}$ a $727^{\circ} \mathrm{C}$, na escala de molalidade ${ }^{12}$.

Para certificar a consistência do resultado deste trabalho e compará-lo ao determinado por Cherginets ${ }^{14}$, correlacionou-se os valores do produto de solubilidade obtidos pelo mesmo método contra a densidade de carga (j) de cada cátion, que pode ser calculada conhecendo-se o raio iônico dos respectivos cátions e admitindo a aproximação de Born (íon como esfera rígida), resultando:

$\mathrm{j}=\frac{\mathrm{z}_{\mathrm{i}} \mathrm{e}_{\mathrm{o}}}{4 \pi \mathrm{r}_{\mathrm{i}}^{2}}$

com $\mathrm{j}=$ densidade de carga superficial; $\mathrm{z}_{\mathrm{i}}=$ valência do íon; $\mathrm{e}_{\mathrm{o}}$ = carga do electron $\left(1,6 \times 10^{-19}\right)$ e $r_{i}=$ raio do íon.

A Figura 3 mostra a linearidade entre os cátions $\mathrm{Mg}^{2+}, \mathrm{Ca}^{2+} \mathrm{e}$ $\mathrm{Sr}^{2+}$, e o mesmo não se observa no trabalho de Cherginets ${ }^{14}$. A dependência linear, isto é, a constante de proporcionalidade entre o produto de solubilidade e a densidade superficial de carga indica que as interações entre soluto e solvente são do tipo eletrostáticas. Quanto ao desvio apresentado pelo íon $\mathrm{Ba}^{2+}$, a possível explicação é o surgimento de interações não coulômbicas, possivelmente há formação de cloro-complexos ${ }^{16-21}$.

\section{CONCLUSÃO}

Quando se compara o resultado encontrado neste trabalho e os existentes na literatura ${ }^{7,8,9,13,14}$, observa-se que a técnica experimental e a substância usada como oxobase determinam a confiabilidade das medidas.

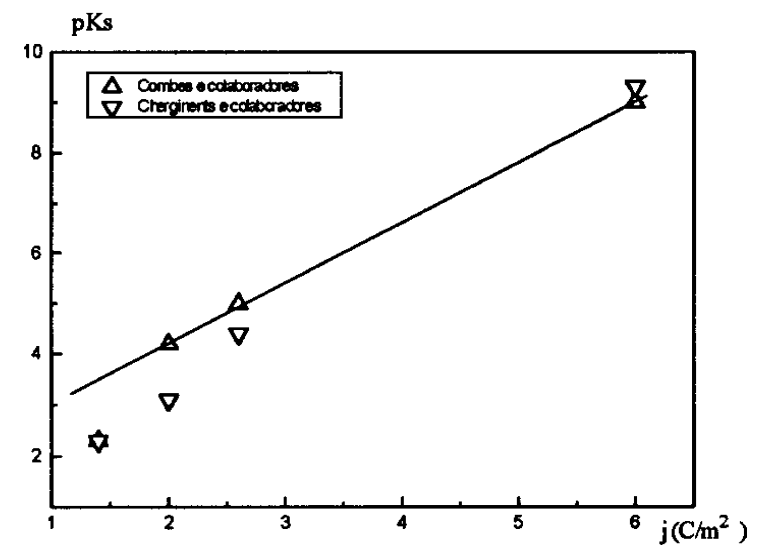

Figura 3. Curva do produto de solubilidade dos alcalino terrosos contra densidade superficial de carga

\section{AGRADECIMENTOS}

Os autores agradecem à Petromisa pelo financiamento deste trabalho.

\section{REFERÊNCIAS}

1. Lux, H.; Z. Elektrochem. 1939, 45, 303. Lux, H.; Naturwiss 1940, 29, 92.

2. Flood, H.; Førland, T.; Acta Chem. Scand. 1947, 1, 592.

3. Harrison, J.; C. R. Acad. Sc. 1955, 241, 298 and 402.

4. Delarue, G.; J. Electroanal. Chem. 1960, 1, 285.

5. Charlot, G.; Tremillon, B.; Les réactions chimiques dans les solvants et les sels fondus, Gauthers-Villars ed., Paris, 1963.

6. Janz, G.; Molten Salts Handbook, Academic Press, NewYork, 1967.

7. Naumann, D.; Reinhard, G.; Z. Anorg. Chem. 1966, $343,165$.

8. Combes, R.; Andrade, F.; C. R. Acad. Sc. 1975, 280, 945, and J. Electroanal. Chem. 1977, 83, 297.

9. Combes, R.; Andrade, F.; Ferreira, H.; Electrochim. Acta 1980, 25, 371 .

10. Combes, R.; Vedel, J.; Tremillon, B.; Anal. Letters 1970, 3, 523.

11. Combes, R.; Vedel, J.; Tremillon, B. C. R. Acad. Sc. Paris, 1971, 273, 1740,; 1972, 275, 199.

12. Combes, R.; Vedel, J.; Tremillon, B.; Electrochim. Acta 1975, 20, 191.

13. Delimarsky, V.; Shapoval, K.; Ukr. Khim. Zh. 1977, 43, 115.

14. Cherginets, V. L.; Khailova, E. G.; Electrochim. Acta 1994, 39, 823 .

15. Blander, M.; Thermodynamic Properties of Molten Salts Solutions, Molten Salts Chemistry, 1964.

16. Druem, D. M.; Espectroscopy of Transition of Metals in Fused Salts, dans le livre de Sundheim: Fused Salts, Mc. Graw-Hill ed. New-York, 1964.

17. Bard, A.; Fused salts systems, in Encyclopedia of electrochemistry of the elements, Marcel Dekker, NewYork, 1976.

18. Tremillon, B.; Proceeding of the first international symposium on molten salts chemistry, Kyoto, Japan, 1983.

19. Bredig, M.; The experimental evidence for complex ions in molten salts, em Molten Salts. G. Mamantov editor, Marcel Dekker, New-York, 1969.

20. Enderby, J.; Biggin, S.; Structural investigations of molten salts by diffraction methods em Advances in molten salts chemistry, G. Mamantov editor, Elsevier, Amsterdam, 1983.

21. Wood, N.; Howe, R.; J. Phys. C. Solid State Physics $\mathbf{1 9 8 8 ,}$ $21,3177$. 